\title{
Las Denuncias Falsas en Casos de Violencia de Género: ¿Mito o Realidad?
}

\section{False Reports in Gender Violence: ¿Myth or Fact?}

\author{
Francisco Pérez Fernández y Beatriz Bernabé Cárdaba \\ Universidad Camilo José Cela, España
}

\begin{abstract}
Resumen. Tras la aprobación de la Ley Orgánica 1/2004 de medidas de protección integral contra la violencia de género, España se ha convertido en el primer país europeo que cuenta con una legislación multidisciplinar de estas características, pues toca todos los aspectos implicados en la materia: educación, prevención y sanción. No obstante, el hecho de que esta ley se adentre en el ámbito de la llamada discriminación positiva y sólo contemple como autor de conductas punibles al varón, entretanto sólo asuma como víctima a la mujer, es cuestión que ha generado un candente debate social e incluso serias cuestiones acerca de su constitucionalidad (Sanmartín, Iborra, García Esteve y Martínez Sánchez, 2010). El debate acerca de la existencia de posibles "denuncias falsas" de maltrato, sin duda, no beneficia a nadie en este controvertido contexto jurídico, político y social. Tratamos, por ello, de profundizar en este ámbito a fin de determinar cuánto de cierto pudiera haber en este asunto que, por el bien mismo de la igualdad, debiera afrontarse y resolverse con decisión y eficacia.
\end{abstract}

Palabras clave: violencia de género; denuncia falsa; Ley 1/2004; igualdad; discriminación.

\begin{abstract}
Following the adoption of the organic law 1/2004 of measures of integral protection based against violence of gender, Spain has become the first European country to have multidisciplinary legislation in effect. This law considers educational criteria, prevention and punishment. The fact that this law is associated to positive discrimination and, only consideres men as the authors of punishable behavior assuming women as the only victims, has generated a heated social debate and even serious questions as to the constitutionality of this law (Sanmartín, Iborra and GarcíaEsteveMartínez Sánchez, 2010). The debate over the existence of possible "false accusations" doesn't help to clarify the real information associated with this phenomena. To do so, the information should be considered in the light of equal rights so that correct and effective decisions can be made.

Keywords: adolescents, restorative, criminal responsibility.
\end{abstract}

Tras la aprobación de la Ley Orgánica 1/2004 de medidas de protección integral contra la violencia de género, España se ha convertido en el primer país europeo que cuenta con una legislación multidisciplinar de estas características, pues toca todos los aspectos implicados en la materia: educación, prevención y sanción. No obstante, el hecho de que esta ley se adentre en el ámbito de la llamada discriminación positiva y sólo contemple como autor de con-

La correspondencia sobre este artículo debe enviarse al autor al email: fperez@ucjc.edu ductas punibles al varón, entretanto sólo asuma como víctima a la mujer, es cuestión que ha generado un candente debate social e incluso serias cuestiones acerca de su constitucionalidad (Sanmartín, Iborra, García y Martínez, 2010).

Es innegable que hasta un tiempo relativamente reciente, y como una parte asumida de la dinámica social, la mujer ha estado sometida a toda clase de vejaciones, insultos y agresiones físicas y psíquicas frente a las que no tenía otra respuesta que el asentimiento. Incluso se consideraba, a menudo, esta situación se servilismo hacia el varón como algo que for- 
maba parte de su entidad y femineidad naturales (Paz y Coronado, 2005). Una manifestación natural de su sumisión al varón en tanto que autoridad paternalista y tutelar, suministrador del dinero para la manutención de la unidad familiar, cúspide del hogar y gestor a quien se debía respeto y obediencia incontestables. No hace mucho tiempo todavía que la mujer, en España, pasaba de la tutela del padre a la del marido, por lo que no podía gestionar propiedades y cuentas bancarias, decidir acerca de las condiciones de su maternidad, o simplemente acceder a un puesto de trabajo, sin la autorización y consentimiento expresos de cualquiera de ambos (Ortiz, 2006). Un hecho tan profundamente introducido en la mentalidad colectiva, y tan cercano, que aún hoy resulta hasta cierto punto comprensible -en estrictos términos de evolución sociocultural- la resistencia de muchos varones educados en aquel patrón -o desde él- a asumir las modificaciones sufridas por el statu quo ético y legal de la mujer en un tiempo relativamente corto. Una razón que, por sí misma, hace necesaria una mayor inversión pedagógica en materia de igualdad.

Los cambios sociales ocurridos tras la caída del régimen franquista y la Transición democrática han procurado, a su vez, transformaciones rápidas en las dinámicas familiares, configurando nuevas tipologías de familia y novedosas relaciones de parentesco. Por supuesto, no se trata de un fenómeno tan solo del momento presente. La historia de la familia, en tanto que institución sociocultural, es larga y está repleta de hitos que la han ido adecuando a la realidad de cada época (Parada, 2010). Sea como fuere, uno de los factores más importantes que están determinando los cambios sociales en la familia española del presente es la incorporación masiva de la mujer al mundo laboral, hecho que ha modificado por completo su estatus jurídico y social en apenas tres décadas. Esta incorporación, como es lógico, ha revolucionado las pautas de conducta, educación y poder en el interior de los núcleos familiares así como los límites de sus funciones socializadoras, pues la mujer, tradicionalmente encargada del hogar y del cuidado y crianza de los hijos, ahora también pasa muchas horas fuera de casa, contribuye activamente al sostenimiento económico de la casa, goza de mayor independencia y se ve obligada a delegar, complementar y matizar sus antiguas funciones.

Inevitablemente, este nuevo estatus sociocultural

Evolución histórica de las personas ocupadas en España, por sexo

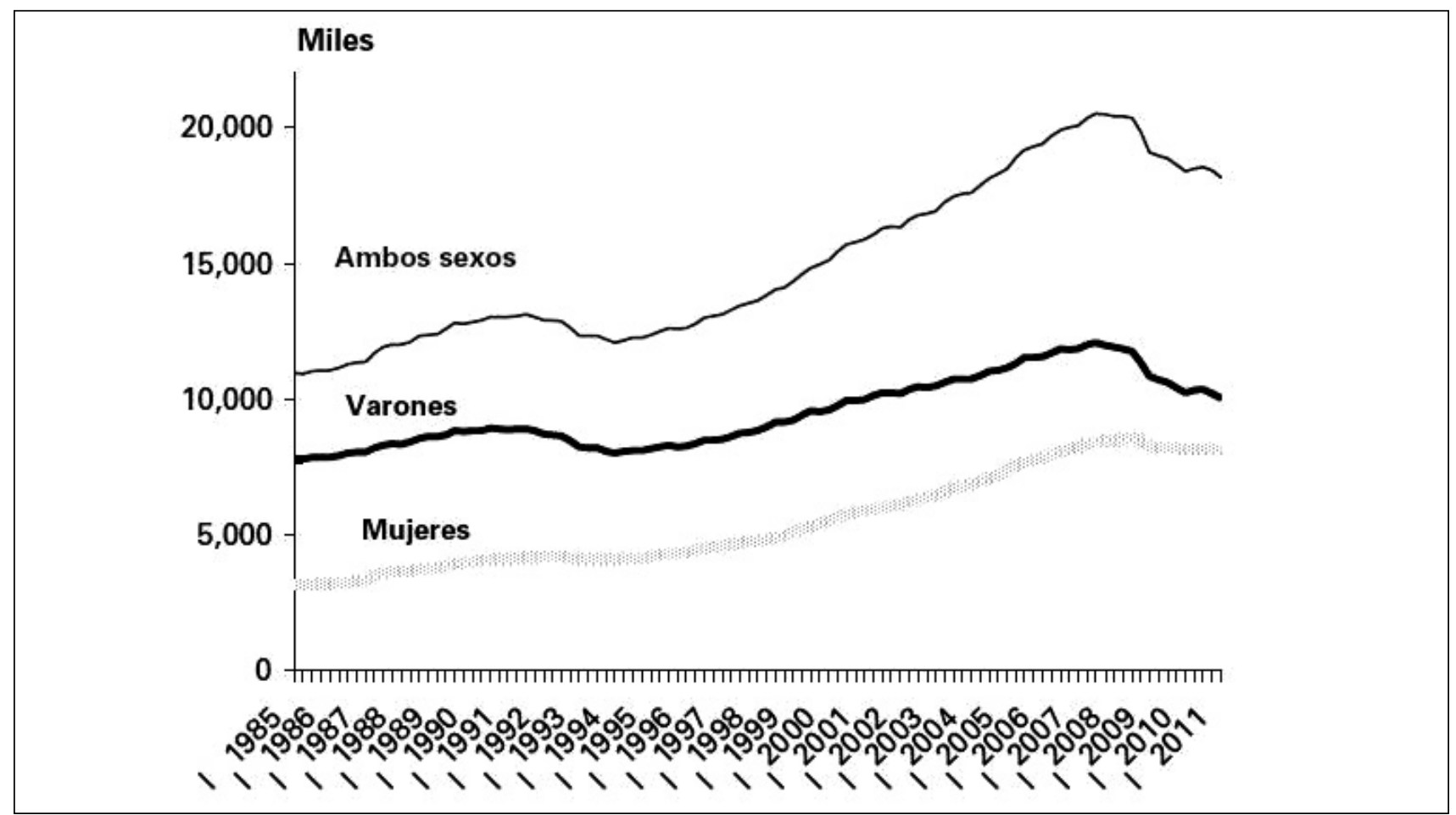

(Fuente: INE, 2011a) 
de la mujer ha motivado que sus problemas tengan mayor visibilidad y que, por tanto, la comunidad y las Autoridades ya no puedan aplazar, soslayar o minimizar asuntos que la afectan directamente como el de la violencia de género. De hecho, la presión social sistemática ha conseguido que los diferentes gobiernos aumentaran progresivamente las ayudas socioeconómicas para las mujeres en situación de riesgo a la par que trataban de combatir el fenómeno por la vía de las reformas legales, la actividad educativa, las campañas de concienciación social y la acción policial. Esta tendencia motivó que los casos de violencia de género -otrora virtualmente invisibles- ocuparan paulatinamente el centro de la vida pública española (Bustelo, López y Platero, 2007).

De tal modo, esta clase de sucesos se ha convertido en lugar común para los medios de comunicación y, por tanto, para la sociedad en general, creándose con ello un acusado estado de alarma social frente al fenómeno. Es por ello que, muy sensibilizados con el problema, tanto jueces como fiscales llegaron al punto de establecer una doctrina favorecedora de la mujer en sus sentencias de separación y divorcio, especialmente en lo relativo a la custodia de los hijos que la pareja pudiera tener en común. Tendencia que más o menos matizada todavía se perpetúa en el presente y, de hecho, en el año 2010 la custodia de los hijos menores fue otorgada a la madre en el $83,2 \%$ de las rupturas matrimoniales, cifra equivalente a la registrada en 2009 , con un 84\% (INE, 2011b).

Esta situación de discriminación positiva no reconocida legalmente, y contra lo pretendido, degeneró en una retroalimentación indeseada de la violencia contra las mujeres que entre los años 2000 y 2006 aumentó en el ámbito doméstico un $15.16 \%$, siendo el tercer país en el que más creció en el susodicho periodo (Sanmartín et al., 2010). Consecuentemente, y a la vista de esta espiral, se llegó a un punto en el que se vieron desbordadas las previsiones jurídicas de lo establecido en el Código Penal y se planteó la necesidad de combatir con eficacia la violencia contra las mujeres a la par que se calibraban y regulaban, adecuadamente, estas situaciones de discriminación positiva.

Ha de quedar claro, antes de continuar, que este trabajo no pretende caer en simplificaciones ni abordar en su integridad los posibles defectos y/o virtudes de la Ley 1/2004, cuestión de enorme actualidad y controversia entre juristas, psicólogos, psiquiatras, educadores y mediadores que excede con mucho nuestros objetivos. Por lo demás, no podemos negar -o minimizar- el terrible hecho de que, tal y como muestran los datos del Instituto Nacional de Estadística $^{1}$, entre el año 2000 y el 2010 murieron 710 mujeres a manos de sus parejas o ex parejas. Una tragedia social deleznable que precisa de un tratamiento psicosocial y criminológico inmediato y a todos los niveles. Pero, y este extremo no es menos cierto, cada vez es más habitual asistir al relato de cómo, escudadas tras esta ominosa realidad, habría personas oportunistas que por motivos muy dispares habrían aprovechado la ola de sensibilización ciudadana y jurídica que ha inundado el cuerpo social, para hacerse con una sentencia favorable $\mathrm{y}$, por supuesto, con las posibles ventajas morales y materiales que ésta lleva aparejadas. Una cuestión de gran impacto sociocultural, aún a caballo entre el mito y la realidad, cuya posible veracidad o falsedad debiera ser-creemos- adecuadamente debatida.

\section{De la discriminación positiva al debate sobre la“denuncia falsa"}

Cuando vio la luz la Ley $1 / 2004$, muy pronto se convirtió en una herramienta para el debate político -en poco tiempo se presentaron ante el Tribunal Constitucional (TC) nada menos que 187 recursos de inconstitucionalidad contra diferentes aspectos del texto- a la par que en un instrumento liberador para aquellos miembros de la judicatura que pudieran sentirse señalados por la presión mediática y popular. Pero también, y de manera colateral, generó un importante debate jurídico-legal al ponerse en tela de juicio elementos básicos del Estado de Derecho como el de la mismísima igualdad ante la ley y el de la presunción de inocencia -hechos que indujeron a muchos jueces a renunciar a la aplicación de los artículos más controvertidos de la Ley 1/2004 hasta que el TC se manifestara sobre los recursos presentados

\footnotetext{
${ }^{1}$ www.ine.es
} 
contra los mismos. Dos tipos de debates controvertidos que han dado lugar a serias polémicas conceptuales, jurídicas e ideológicas (Barrere, 2003), que han sido zanjadas en el plano legal en más de un sentido por las resoluciones del TC, pero no así en lo relativo a la discusión política.

Cabría pensar que la entrada en vigor de la Ley 1/2004 habría supuesto, en tanto que persecución directa de estas actividades criminales contra las mujeres, un descenso radical de la violencia contra ellas, pero nada más alejado de la realidad. Como se ha indicado a través de diferentes estudios de las cifras criminales españolas: "Los delitos contra la vida, la integridad y la libertad de las personas, mostraron una curva de marcada estabilidad entre los años 2000 y 2002. Posteriormente aumentaron de manera progresiva -e incluso rápida- pasando de 1.62 por cada mil habitantes en 2002, a 2.70 en 2009. Aquí se pone de manifiesto demanera clara la importancia para la medición de la criminalidad que ha significado la Ley Integral Contra la Violencia de Género pues del mismo modo que ha provocado un aumento de las denuncias y detenciones en este ámbito [...], parece no haber tenido una incidencia clara en el descenso de la violencia contra las mujeres" (Pérez-Fernández, Pérez-Abellán, AntolínezMerchán, Cabrera y Siegl, 2012; p. 19). De hecho, el incremento de denuncias por malos tratos interpuestas por mujeres hacia sus parejas o exparejas no sólo no ha disminuido, sino que ha seguido una tendencia de incremento constante. Así, mientras en que 2003 se interpusieron 50.090 denuncias, en 2007, tres años después de la entrada en vigor de la Ley $1 / 2004$, ya se contabilizaron $126.293^{2}$ (Pérez del Valle, 2009).

Mucho se ha discutido a costa de este efecto rebote, inexplicable si tenemos en cuenta el hecho de la ya reseñada concienciación sociopolítica en torno al problema, pero no parece tan extraño cuando se tiene en cuenta, entre otras cosas, que la desventaja del hombre ante la mujer en este particular jurídico aunada a una falta endémica de políticas preventivas, ha involucionado hacia situaciones de fuerte

\footnotetext{
${ }^{2}$ Este inusitado incremento en las cifras podría tratar de explicarse a través del impacto generado entre la población femenina por las campañas de concienciación a favor de la denuncia de las situaciones de maltrato, pero lo cierto es que tal aseveración no deja de resultar especulativa por cuanto se trata un extremo no establecido científicamente.
}

carga emocional -fuente de nuevas violencias- que han limitado el efecto de la legislación en sí misma conduciendo a muchos profesionales a poner en tela de juicio alguno de sus aspectos centrales en la medida que generadores de contradicciones irresueltas y en absoluto baladíes: "La construcción social del binomio formado por agresor y víctima atribuye el uso y el monopolio de la violencia a los hombres y la pasividad a las mujeres. En la medida en que se asigna a las mujeres una posición fija de pasividad, el Estado asume el papel activo de protección de éstas - pretendidamente desvalidas y no violentas - frente a los hombres violentos. La relación que lasmujeres tienen con la violencia se limita al papel de sufrirla, lo cual las colocaen una posición infantil y, por ello, dependiente de la actuación del Estado. Desde esta lógica, no se trata de sacar a la mujer de esta posición o, como mínimo, cuestionarla, sino de castigar al agresor que se excede" (Coll-Planas, García-Romeral, Mañas-Rodríguez y NavarroVaras, 2008, p. 201).

No en vano, y a pesar de la múltiples ayudas, esfuerzos, ventajas, organismos, campañas y beneficios que la legislación y las instituciones han procurado a la mujer para su defensa, y que se obtienen "con la simple interposición de la denuncia en unos casos o del dictado de la orden de protección en otros, sin necesidad de esperar al menos a la sentencia condenatoria en los juicios rápidos [...], lo cierto es que de los datos oficiales publicados puede llegarse a la conclusión de que la mujer no ha sido protegida adecuadamente con esta Ley [1/2004] en el ámbito penal y no se han cumplido ni mucho menos las expectativas esperadas (Pérez del Valle, 2009).

Estas curiosas asimetrías entre hombre y mujer, no exclusivas de la legislación española pero si acentuadas por una ley tan específica -y peculiar- como la $1 / 2004$, que por su propia idiosincrasia tiende a otorgar a la mujer el inevitable papel de víctima y al hombre el ineludible de verdugo ${ }^{3}$, han sido someti-

\footnotetext{
${ }^{3}$ Un sesgo curioso que contraría buena parte de la literatura científica internacional, así como los resultados obtenidos por muchas de las macroencuestas sobre criminalidad realizadas en otros países. Tales documentos avalan un claro sentido de bidireccionalidad en lo relativo a la violencia entre sexos, ya sea en el ámbito intra o extrafamiliar, estableciendo niveles parejos de conflictividad y virulencia para ambos sexos (así por ejemplo, Feather, 1996; Mihalic y Elliott, 1997; Archer, 2002; Ehrensaft, Moffit y Caspi, 2004, y otro largo etcétera).
} 
das a profunda crítica por cuanto, se sostiene desde los entornos más críticos, degenerarían en picarescas como la de las denuncias falsas de maltrato a la pareja o de abuso sexual sobre los hijos en los procesos de separación y divorcio. Un fenómeno que pese a no haber sido materia para un estudio exhaustivo $-\mathrm{o}$ al menos claro- en España, tampoco es en caso alguno nuevo, tratándose de un hecho ampliamente registrado en la literatura anglosajona desde hace décadas (así, y por citar dos conocidos, Kaplan y Kaplan, 1981 o Ross y Blush, 1990). Estos estudios internacionales manifiestan que para muchas mujeres denunciar a sus exparejas en relación a abusos sobre los hijos -fenómeno conocido popularmente como bala de plata - es contemplado como un recurso "infalible" que consigue la imposición automática de medidas cautelares y la permanente sombra de la sospecha sobre el varón, ante los tribunales, durante el resto de proceso. A tal respecto, la literaturaha establecido, tras un profuso estudio de estas denuncias falsas por abusos sobre los hijos presentadas en tribunales estadounidenses, un perfil tipo de las mismas (Wakefield y Underwager, 1990):

1. La denuncia siempre aparece tras la separación, y en el contexto de las acciones legales.

2. A menudo existe un historial de disfunciones familiares relacionadas con otros conflictos irresueltos.

3. El perfil de la acusadora es el de una persona enfadada, a la defensiva y autojustificativa que podría padecer algún tipo de trastorno de personalidad (límite o histriónico por lo general).

4. El perfil del acusado es el de una persona pasiva, culta y carente de los atributos habituales del macho.

5. La supuesta víctima de los abusos responde al perfil de una niña menor de ocho años.

6. La denuncia siempre es presentada por el progenitor -la madre por lo común- que tiene la custodia efectiva o provisional de la supuesta víctima.

7. La madre acusadora siempre acude con el niño a un "experto" cuyo testimonio confirma el abuso e identifica al padre como abusador.

8. Habitualmente, el tribunal reacciona al testimonio experto limitando o cesando las visitas del padre.
Debe señalarse, por lo demás y en todo caso, que los estudios indican que este problema no está necesariamente relacionado con variables como el sexo del denunciante, pese a que afecte en mucha mayor medida a las mujeres en tanto que principales beneficiarias jurídicas de la custodia legal de los hijos durante los procesos de separación. Por otra parte, diversos estudios han mostrado que la denuncia falsa de abusos o malos tratos durante las etapas previas -o intermedias- de los procedimientos judiciales guardarían una relación más o menos clara con las manifestaciones del llamado síndrome de alienación parental o SAP (Bermúdez, 2009).

\section{Denuncia falsa: ¿mito o realidad?}

Entretanto la literatura científica anglosajona muestra una amplia cantidad de trabajos relacionados con las denuncias falsas de agresión sexual expresadas por niños y adolescentes, el problema que nos ocupa es prácticamente invisible en relación a la mujer adulta y las supuestas acusaciones falsas de malos tratos. Más aún, en España el asunto está prácticamente ausente de la literatura científica lo cual nos habla de un claro sesgo de autocensura en la investigación que afecta, incluso, a la cuantificación y consideración social y jurídica del problema. Así, ninguna de las fuentes estadísticas habituales en materia judicial (Ministerio de Justicia, Ministerio del Interior y Consejo General del Poder Judicial) ofrece datos más o menos claros en este sentido pues ni tan siquiera los cuantifican con rigor y, de ofertarlos, se presentan al público ya elaborados. Así por ejemplo, y en una línea informativa que ya es habitual, el Observatorio contra la Violencia Doméstica y de Género (órgano del CGPJ), establece que en el segundo cuatrimestre del año 2011 se produjo un total de 40,9\% de sentencias absolutorias, pero no se aclara cuántas de ellas podrían ser consideradas "falsas" o "dudosas".

Profundicemos en los escasos datos de que se disponen a fin de subrayar el "lado oscuro" de las cifras del que prácticamente nadie habla: Alrededor de la cuarta parte de los hombres denunciados por violencia de género nunca son condenados (Pérez del Valle, 2009). Así, de los 480.663 procedimien- 
tos abiertos contra varones desde la entrada en vigor de la Ley 1/2004 hasta el año 2008, 95.328 (un 19.83\%) se sobreseyeron provisionalmente y nunca llegaron a juicio; 12.455 (2.59\%) fueron absueltos tras los pertinentes juicios de faltas celebrados en los juzgados de violencia contra la mujer; $24.267(5.04 \%)$ resultaron absueltos por los juzgados de lo penal y $87(0.018 \%)$ fueron absueltos en las audiencias provinciales. En total, 132.137 varones -el $27.49 \%$ de los denunciados- jamás resultaron condenados sin que ello les evitara los pertinentes perjuicios sociales, morales y jurídicos que les produjo verse envueltos en semejante situación, y de los cuales nunca fueron resarcidos (CGPJ, 2008). Podría argumentarse que este exagerado número de sobreseimientos pudiera tener que ver con el hecho de que muchas mujeres retiren sus denuncias, pero no existe coincidencia a este respecto en los datos pues la ratio de renuncias, ofrecida por la misma fuente, se cifra en torno al $10 \% \mathrm{y}$, por lo demás, la legislación establece que los procedimientos de violencia de género sigan adelante con independencia de que la denunciante se retracte de sus intenciones.

La escasa profundización en los motivos y efectos de las cifras que venimos desgranando a lo largo de este trabajo, y que apenas si rascan la corteza de una realidad repleta de opacidades, ha generado un claro sesgo informativo que ha calado hondamente en la opinión pública y que ha servido para que, desde diferentes sectores pro-derechos de la mujer, se defienda con cierta solvencia estadística la idea de que las denuncias falsas son un simple mito (Balseiro, 2008) que, por tanto, no debiera ni tan siquiera ser considerado. Un backlash ideado por la "cultura machista" para mantener su opresión sobre la mujer puesto que si no hay dato empírico fiable y contrastado, no existe problema real. Justamente $-\mathrm{y}$ de suerte paradójica- la misma clase de argumento que se utilizó durante siglos para ocultar hechos incuestionables como la opresión, el sometimiento y la violencia contra las mujeres. Más aún, y dado que no se encuentra tipificado en las guías de los trastornos mentales más utilizadas (CIE y DSM), este tipo de planteamientos niega radicalmente la existencia de supuestas patologías como el SAP, u otras similares, y las considera acientíficas y parte de esa con- traofensiva del establishment machista (Escudero, Aguilar y Cruz, 2008), por lo que advierte de los riesgos inherentes a que las resoluciones judiciales puedan verse afectadas por ellas.

El problema objetivo, sin embargo, es otro bien diferente: la denuncia falsa, de poder constatarse mediante estudios empíricos de calidad científica contrastada, no sólo perjudicaría al varón en tanto que receptor pasivo de la misma, sino también a la mujer realmente maltratada en la medida que práctica perniciosa que, de generalizarse, podría terminar por someterla a la desconfianza pública y jurídica. Así lo advertía, no sin tibieza, la propia Fiscalía General en 2006 al advertir que el uso indebido de la Ley 1/2004 "no es cierto, aunque aparezcan casos aislados como en cualquier otra actividad delictiva, casos que con más empeño habrá que dilucidar por el daño que se hace a las mujeres sometidas a malos tratos físicos o psicológicos que se ven subordinadas a una duda irracional e injusta" (Memoria de la Fiscalía General del Estado, 2006, p. 357).

No obstante, dentro de esta corriente negacionista -o mitificadora- de la denuncia falsa, y que parece tener más un carácter ideológico que propiamente científico, comienzan a aparecer voces discordantes, sobre todo fuera de España. Cada vez son más las feministas norteamericanas, adscritas a ámbitos académicos, que consideran que el grueso del movimiento feminista incurre en un falseamiento sistemático de la realidad al convertirse, más que en un movimiento de lucha por los derechos de la mujer, en un movimiento de victimización de la misma."Señalan también, que gran parte de la investigación feminista carece de rigor científico y está imbuida, ydemasiado contaminada, por la ideología feminista. Afirman que estas deficiencias decalidad se trasladan a los estudios de mujer de las universidades, cuyos contenidos sonlimitados por la censura de la corrección política feminista que opera en ellos. Culpan al feminismo de haber creado un estado de alerta y crispación con la extensiónde la definición de los conceptos de acoso y agresión sexual más allá del sentido común”(León, 2009).

El hecho es que los supuestos casos de "denuncia falsa", una vez establecido este contexto complejo en el que no pueden gozar de atención y cobertura 
dentro del discurso oficial, quedan finalmente reducidos al espacio de los foros y los chats de internet, o a los informes de actualidad de los medios de comunicación, pues solo allá las pretendidas víctimas de esta estrategia acusatoria parecen recibir oídos, apoyo, y encontrar una vía para la difusión de su problema. Lo cierto es que los impulsores de las nuevas asociaciones de afectados por las denuncias falsas de maltrato que empiezan a consolidarse en España estiman que la desigualdad de trato con respecto a la mujer ante los tribunales está sirviendo para que se silencien otras estadísticas ominosas, como la de la cantidad de hombres que sufren la violencia intrafamiliar ${ }^{4}$.

De hecho, hemos de reconocer como autores de este trabajo que, frente a lo que sucede en el caso de la violencia del hombre hacia la mujer, en el que las estadísticas son profusas, profundas y generadas en múltiples fuentes, nos ha sido verdaderamente difícil encontrar, dentro del contexto español, datos fidedignos que discurran en el sentido contrario -O que simplemente lo traten. Si ubicamos esta ausencia en sintonía con la realidad que se describe en los estudios internacionales ${ }^{5}$ nos enfrentamos, evidentemente, a fundadas sospechas sobre el silenciamiento psicosocial, cultural, político y jurídico de un problema y, a la par, de una muestra más del interesado control de las estadísticas criminales que se ejerce por parte de los poderes públicos y que ya ha sido denunciado por los investigadores en relación a otros ámbitos y temáticas delictivas (Aebi y Linde, 2010).

\section{Conclusión: ¿Hay un perfil en la denuncia falsa?}

La única vía material de acceso a este problema que nos ha permitido el estado de la cuestión anteriormente descrito ha sido el análisis de las sentencias del Tribunal Supremo lo cual, al menos, aclara el panorama y permite penetrar con eficiencia en el

\footnotetext{
${ }^{4}$ Resulta muy esclarecedora en este sentido la revisión del llamado "Informe Iceberg", presentado por un grupo independiente de padres separados ante la Comisión Mixta de los Derechos de la Mujer del Senado de España, durante la sesión del 25 de junio de 2001 [puede consultarse en http://www.conpapaymama.org/documentos/ice_mini. pdf; tomado en marzo de 2012].

${ }^{5}$ Véase nota 3.
}

problema de la tipificación de la denuncia falsa frente a la que no lo es ${ }^{6}$. Más aún, este análisis muestra que existiría una caracteriología más o menos común a este tipo de denuncias que se encuentra en cierta sintonía - no en pie de igualdad pues no tratamos el mismo problema específico-, especialmente cuando la violencia aparece en contextos de divorcio y/o separación y existen hijos de por medio, con la descrita en la literatura en torno al ya citado SAP (Oropeza, 2007).

Tampoco es desdeñable, por cierto, el parecido que la posible denuncia falsa en el campo de los malos tratos mostraría con la que puede encontrarse en otros entornos jurídicos, como el del acoso laboral o los abusos sexuales, y ello podría deberse, aventuramos, al hecho de que el falso denunciante, sea cual fuere su naturaleza y por su propio interés litigante, busca en última instancia el beneficio personal, el perjuicio de la parte -o partes-denunciada, o bien ambas cosas (Kvitko, 2006).

Tomando como punto de corte el año 2004, momento en el que entra vigor la Ley $1 / 2004$, encontramos que:

- Parece existir una importancia elevada del factor económico. De hecho, la mayor parte de las denuncias falsas tienden a darse con mayor asiduidad entre la población de nivel económico medio-bajo, por lo común, la parte denunciante suele ser la de mayor insolvencia.

- Se muestra tópico en aquellos casos en los que la denuncia parece falsa que la parte denunciante muestre un elevado grado de querulancia, insistiendo más en el ataque sistemático hacia la personalidad de la parte denunciada que en el detalle de los hechos que se denuncian.

- La denuncia falsa parecería insistir más en el dolo psicológico que en el maltrato físico. De hecho, sería más fácilmente identificable por el defecto y la circunstancialidadde lo denunciado que por el exceso, ya que se apoyaría más en la acumulación masiva de indicios sospechosos que en la aportación de datos -o episodiosconcretos y relevantes de malos tratos. Más aún, parece darse una tendencia hacia la omisión expresa de cualquier detalle que pueda ser

\footnotetext{
${ }^{6} \mathrm{http}: / /$ noticias.juridicas.com.
} 
tomado ocasionalmente como benéfico o exculpatorio para con la parte denunciada.

- No es raro que la falsa denuncia -algo habitual en estos casos- sea instigada por una tercera persona (familiar, amigo, conocido) afín a la parte denunciante. Por ello es relevante, en caso de que existan sospechas acerca de la falsedad de la denuncia, prestar atención a elementos externos como la calidad del testimonio de la víctima, su repetitividad, la cantidad de pruebas materiales que se aporten, y la mayor o menor circunstancialidad de aquello que se denuncia.

En lo relativo al diseño de un perfil psicológico del falso denunciante por malos tratos, encontramos elementos que, a menudo, son tópicos en cualesquiera otras tipologías de falsos denunciantes, así como detalles testificales que han de ser tenidos en cuenta -y examinados llegado el caso- por los profesionales implicados a fin de no incurrir en errores (Maqueda, 1999):

Personalidad paranoide y disocial (criterio CIE10). Esta se detecta tanto por su insistencia contumaz en la acusación (querulancia o litigancia activa), como por su amoralidad en la exigencia de una justicia que la propia parte denunciante no respeta. De hecho, y precisamente por ello se muestra muy resistente y ansioso frente a las vistas orales y careos, el falso denunciante incurre a menudo en delirios conspiratorios e intolerancia extrema hacia las opiniones y/o criterios discrepantes. Por ello, cuando se sospecha una falsa denuncia se deben considerar los expedientes médicos, psicológicos y psiquiátricos que puedan existir, especialmente si la denuncia atañe a casuística relacionada con abusos sexuales. También pueden ser muy valiosos los testimonios de las personas cercanas -familiares, compañeros de trabajo y etcétera- de quien se sospecha fundadamente que denuncia en falso.

Si la denuncia tiene que ver con abusos sexuales, especialmente cuando estos involucren a niños, es importante saber cómo se obtuvo la información acerca de los mismos pues, contrariamente a lo que suponen muchos psicoterapeutas, la información obtenida bajo hipnosis o en grupos de apoyo a menudo no es fiable. Tampoco es raro que sucedan situaciones de recuerdo recobrado, que habitualmente son falsos como se muestra en la literatura, si las preguntas del terapeuta son demasiado inductoras o sugerentes (Loftus y Ketcham, 1994; Yapko, 1994). También se debe ser cuidadoso con los testimonios de menores en el caso de que la parte denunciante insista con obstinación en que testifiquen.

Deben examinarse, si aparecen, los motivos por los que el denunciante expresa resistencias -a menudo fuertes- a que otros expertos ajenos a su parte ofrezcan una segunda opinión acerca de su caso en concreto.

Lamentablemente, con las limitaciones de la literatura y de los datos existentes en el panorama científico español en el momento presente, resulta harto complejo profundizar más en esta cuestión que, pese a su enorme actualidad, solo parece admitir determinados discursos mostrándose, al parecer, inmadura para evolucionar hacia otras vías de conocimiento.

Y sin embargo, más allá de la obvia discriminación a la que se somete al varón desde las premisas de la legislación actual -lo cual ya sería por sí mismo una razón más que suficiente para una investigación sistemática-, el tema merece estudio y consideración en atención a la propia mujer maltratada que, como ya se ha indicado, puede terminar por ver menoscabada su defensa a causa del uso arbitrario que pudiera hacerse de una legislación que, siendo mejor o peor, se diseño para protegerla. No olvidemos con cuánta facilidad aquellos mismos discursos que tanto esfuerzo -formal y material- cuesta introducir en la sociedad pueden verse suprimidos por el crecimiento de corrientes de opinión reactivas a ellos.

A menudo se dice que hace falta aún mucho esfuerzo pedagógico y material para que la sociedad y la cultura evolucionen hacia la igualdad efectiva, a todos los niveles, entre hombres y mujeres. Y es cierto. Pero tampoco es menos verdad que determinadas actitudes sociopolíticas e informativas, carentes de fundamento científico real y de corte limitante, impiden que se penetre con eficacia en el epicentro de esos mismos problemas que se pretende combatir y erradicar. Hace falta pues que, siguiendo el ejemplo de otros países de nuestro entorno, alcancemos la altura de miras suficiente como para poder elevarnos con eficacia sobre los prejuicios, las visiones sesgadas, las sombras y las medias verdades. El conocimiento, y conviene recordarlo de vez en cuando, sólo es perjudicial para quien teme a la verdad. 


\section{Referencias}

Aebi, M. F. y Linde, A. (2010). El misterioso caso de la desaparición de las estadísticas policiales españolas. Revista Electrónica de Ciencia Penal y Criminología. Recuperado de http://criminet. ugr.es/recpc

Archer, J. (2002). Sex differences in physically aggressive acts between heterosexual partners: A meta-analytic review. Aggression and Violent Behavior, 7, 213-351.

Balseiro, A. (2008). La imagen como reflejo de la violencia y como control social. Amador Carretero, $\mathrm{M}^{\mathrm{a}}$.P. (Dir.); Robledano Arillo, J.; Ruiz Franco $\mathrm{M}^{\mathrm{a}}$.R. (Coords.), Actas del Primer Congreso Internacional sobre Imagen, Cultura y Tecnología (56-68). Madrid: Universidad Carlos III.

Barrere, M. A. (2003). Igualdad y discriminación positiva. Un esbozo de análisis teórico-conceptual. Cuadernos Electrónicos de Filosofía del Derecho, 9. Recuperado de http://www.uv.es/ cefd/Index_9. htm

Bermúdez, M. (2009). El síndrome de alienación parental como elemento valorativo de violencia familiar psicológica. Revista Electrónica Aportes Andinos, 25. Recuperado de http://www.uasb. edu.ec

Bustelo, M., López, S. y Platero, R. (2007). La representación de la violencia contra las mujeres como un asunto de género y un problema público. En M. Bustelo y E. Lombrado (Coords.), Políticas de igualdad en España y en Europa (pp. 67-96). Madrid: Cátedra.

Coll-Planas, G., García-Romeral, G., Mañas-Rodríguez, C. y Navarro-Varas, L. (2008). Cuestiones sin resolver en la Ley Integral de Medidas Contra la Violencia de Género: Las distinciones entre sexo y género, y entre violencia y agresión. Papers. Revista de Sociología, 87, 187-204.

Consejo General del Poder Judicial (2008). Datos estadísticos judiciales en la aplicación de la L.O. 1/2004. Resumen de los tres primeros años de Ley Integral. Recuperado de http://www.poderjudcial. es

Consejo General del Poder Judicial (2011). Datos de denuncias, procedimientos penales y civiles registrados, órdenes de protección solicitadas en los juzgados de violencia sobre la mujer (JVM) y sen- tencias dictadas por los órganos jurisdiccionales en esta materia en el segundo trimestre del año 2011. Recuperado de http://www.poderju dicial.es Ehrensaft, M. K., Moffitt, T. E. y Caspi, A. (2004). Clinically abusive relationships in an unselected birth cohort: men's and women's participation and developmental antecedents. Journal of $A b$ normal Psychology, 113, 258-270.

Escudero, A., Aguilar, A. y Cruz, J. de la (2008). La lógica del Síndrome de Alienación Parental de Gardner (SAP): "terapia de la amenaza". Revista de la Asociación Española de Neuropsiquiatría, 38, 283-305.

Feather, N. T. (1996). Domestic violence, gender and perceptions of justice. Sex Roles, 35, 507-519.

Instituto Nacional de Estadística (2011a). Encuesta de Población Activa (EPA). Primer trimestre de 2011. Notas de Prensa, 29 de abril.

Instituto Nacional de Estadística (2011b). Estadística de nulidades, separaciones y divorcios, año 2010. Notas de prensa, 19 de septiembre.

Kaplan, S. L. y Kaplan, S. J. (1981). The child's accusation of sexual abuse during a divorce and custody struggle. The Hillside Journal of Clinical Psychiatry, 3, 81-95.

Kvitko, L. A. (2006, mayo). Errores periciales y falsas denuncias de abuso sexual infantil. Revista de la Escuela de Medicina Legal, 39-42.

León Mejía, A. (2009). ¿Disidencia dentro del feminismo? Revista Internacional de Sociología, 67, 559-588.

Loftus, E. F. y Ketcham, K. (1994). The myth of repressed memory.New York: St. Martin'sPress.

Maqueda Abreu, Ma . L. (1999). Acusación y denuncias falsas. Valencia: Tirant lo Blanch.

Memoria de la Fiscalía General del Estado (2006). Fiscales de Sala Delegada contra la Violencia sobre la Mujer. Recuperado dehttp://www. fiscal.es

Mihalic, S. W., y Elliot, D. (1997). A social learning theory model of marital violence. Journal of Family Violence, 12, 21-46.

Oropeza Ortiz, J. L. (2007). Síndrome de alienación parental. Actores protagonistas. Revista Internacional de Psicología, 8, Recuperado de http://psicologiarevista.99k.org/numeros.html

Ortiz Heras, M. (2006). Mujer y dictadura franquista. Aposta. Revista de ciencias sociales, 28 . Re- 
cuperado de http://www.apostadigital.com/ revistav3/hemeroteca/ortizheras.pdf

Parada Navas, J. L. (2010). La educación familiar en la familia del pasado, presente y futuro. Educatio Siglo XXI, 28, 17-40.

Paz Rebollo, M. A. y Coronado Ruiz, C. (2005). Mujer y formación profesional durante el franquismo. NO-DO, 1943-1975. Pandora. Revued' EtudesHispaniques, 5, 133-145.

Pérez del Valle, F. (2009 enero). La Ley de Violencia de Género: Lo que no se cuenta de la misma. Locus Apellationis: Boletín Informativo del Ilustre Colegio de Abogados de León, 54. Recuperado de http://www.ical.es/locus/54/index.html

Pérez-Fernández, F., Pérez-Abellán, F., AntolínezMerchán, P., Cabrera-Rodríguez, E. C. y Siegl, D. (2012). Proyecto Ángeles Custodios. Índice de Seguridad Ciudadana de la Universidad Camilo
José Cela. Villanueva de la Cañada (Madrid): Universidad Camilo José Cela.

Ross, K. y Blush G. (1990). Sexual abuse discriminators on the divorced or divorcing family.Issues in Child Abuse Accusations, 2, 1-6.

Sanmartín, J., Iborra, I., García Esteve, Y. y Martínez Sánchez, P. (2010). III Informe internacional. Violencia contra la mujer en las relaciones de pareja. Estadísticas y legislación. Valencia: Centro Reina Sofía Para el Estudio de la Violencia.

Wakefield, H. y Underwager, R. (1990).Personality characteristics of parents making false accusations of sexual abuse in custody disputes. Issues in Child Abuse Accusations, 2, 121-136.

Yapko, M. D. (1994). Suggestions of abuse: True and false memories of childhood sexual trauma. New York: Simon \& Schuster.

Manuscrito recibido: 08/03/2012 Revisión recibida: 08/05/2012 Aceptado: 11/05/2012 